\title{
Top quark properties and mass measurements with the ATLAS detector
}

\author{
Matteo Negrini \\ On behalf of the ATLAS Collaboration \\ INFN - Sezione di Bologna - Via Irnerio 46 - 40126 Bologna - Italy
}

\begin{abstract}
Highlights on recent measurements of top quark properties in ATLAS, using $p p$ collision data at $\sqrt{s}=8 \mathrm{TeV}$ and $13 \mathrm{TeV}$, are presented. The measurements of the top quark polarization and spin correlation coefficients, the $W$ boson helicity fractions, the structure of the $W t b$ vertex, the associated production of a $t \bar{t}$ pair with a vector boson or a photon, and the top quark mass are all in agreement with the Standard Model expectations.
\end{abstract}

Keywords: ATLAS, top quark, spin, associated production, mass

\section{Introduction}

The top quark, the heaviest fundamental particle in the Standard Model (SM) of particle physics, has a lifetime shorter than the hadronization timescale and gives the unique opportunity to study the properties of a "bare" quark. The huge top quark samples collected by the experiments at the Large Hadron Collider (LHC) at CERN, and the availability of next-to-leading order (NLO) or next-to-next-to-leading order (NNLO) predictions for some of the top quark properties, allow to perform precision tests of the SM.

The measurements reported here use events collected by the ATLAS experiment [1] in $p p$ collisions at the center of mass energy $\sqrt{s}=8 \mathrm{TeV}$ and $13 \mathrm{TeV}$ in which a $t \bar{t}$ pair is produced. Since the top quarks decay almost entirely in a $W b$ pair, the top quark decay is defined as "hadronic" or "leptonic" depending on the subsequent decay of the $W$ boson, in a quark anti-quark pair or in a lepton ${ }^{1}$ neutrino pair, respectively.

When both top quarks decay leptonically ("dilepton" channel) the presence of two leptons and two $b$-tagged jets produce a clean signature that allows the selection of high purity samples, however the leptonic branching

\footnotetext{
${ }^{1}$ The word "lepton" refers to electrons and muons throughout this report.
}

fraction of the top quark is smaller than the hadronic one and the presence of two neutrinos in the final state requires kinematic assumptions to infer the complete kinematics of the $t \bar{t}$ system. The channel with both top quarks decaying hadronically ("all-hadronic" channel) is the one with the largest branching fraction and the presence of 6 jets in the final state allows to close the event kinematics, but the selected sample unavoidably contains a large fraction of multi-jet QCD that are produced with a large cross-section at the LHC. A compromise is represented by the "lepton+jets" channel, where one of the top quarks decays hadronically and the other leptonically, which allows to select high purity samples due to the presence of the lepton and two $b$-tagged jets and allows to close the event kinematics by inferring the unmeasured longitudinal momentum of the neutrino using the $W$ mass constraint.

\section{Top quark polarization and $t \bar{t}$ spin correlation}

Top quark pairs are produced unpolarized at the LHC, except for small corrections at the per-mil level, however the spins of $t$ and $\bar{t}$ are correlated and the information is directly transferred to their decay products since the decorrelation induced by the hadronization does not occur. The spin correlation depends on the production 
mechanism, so new physics phenomena could alter the correlation expected in the SM because of additional production mechanisms that may come into play.

The ATLAS experiment performed the measurement of 15 spin observables using $20 \mathrm{fb}^{-1}$ of $\sqrt{s}=8 \mathrm{TeV}$ collision data using the dilepton channel [2].

Three orthogonal spin quantization axes are defined: $k$ given by the $t$ direction in the $t \bar{t}$ rest frame, $n$ transverse to the production plane defined by the $t$ direction and the beam axis, and $r$ orthogonal to the previous axes. The normalized double-differential cross-sections for $t \bar{t}$ production and decay is described as:

$$
\begin{aligned}
& \frac{1}{\sigma} \frac{d^{2} \sigma}{d \cos \theta_{+}^{a} d \cos \theta_{-}^{b}}=\frac{1}{4}\left(1+B_{+}^{a} \cos \theta_{+}^{a}\right. \\
& \left.\quad+B_{-}^{b} \cos \theta_{-}^{b}-C(a, b) \cos \theta_{+}^{a} \cos \theta_{-}^{b}\right),
\end{aligned}
$$

where $\theta$ are the angles between one of the 3 spin quantization axes (indicated with $a, b$ ) and one of the two leptons, the + and - sign refer to $t$ and $\bar{t}$, respectively, $B^{a}=3\left\langle\cos \theta^{a}\right\rangle$ are 6 polarization coefficients, and $C(a, b)=-9\left\langle\cos \theta_{+}^{a} \cos \theta_{-}^{b}\right\rangle$ are 9 spin correlation coefficients.

Reconstructed differential cross-sections are corrected at parton level in the full phase-space, to allow comparison with theoretical calculations, and at particle level in a fiducial volume, to allow comparison with Monte Carlo (MC) simulation predictions. In the latter case, particle level objects are reconstructed from stable particles produced by the event generator. The fiducial region is defined by applying a selection on particle level reconstructed objects to reproduce as closely as possible the event selection applied to objects reconstructed at the detector level, in order to minimize the theoretical extrapolations.

All measured coefficients are in good agreement with the SM theoretical expectations, within uncertainties. The largest contribution to the uncertainty may vary for the different measured parameters, in general it is due to $t \bar{t}$ signal modeling and accounts for approximately $80 \%$ of the total systematic uncertainty.

\section{3. $W$ polarization in $t \bar{t}$ events and $W t b$ vertex anomalous couplings}

The polarization of a $W$ boson, produced as a real particle in the decay of a top quark, can be left-handed, right-handed, or longitudinal. The corresponding helicity fractions are determined by the $W t b$ vertex structure and are computed in the SM at the NNLO in QCD to be $F_{L}=0.311 \pm 0.005, F_{R}=0.0017 \pm 0.0001$, and $F_{0}=0.687 \pm 0.005[3]$.

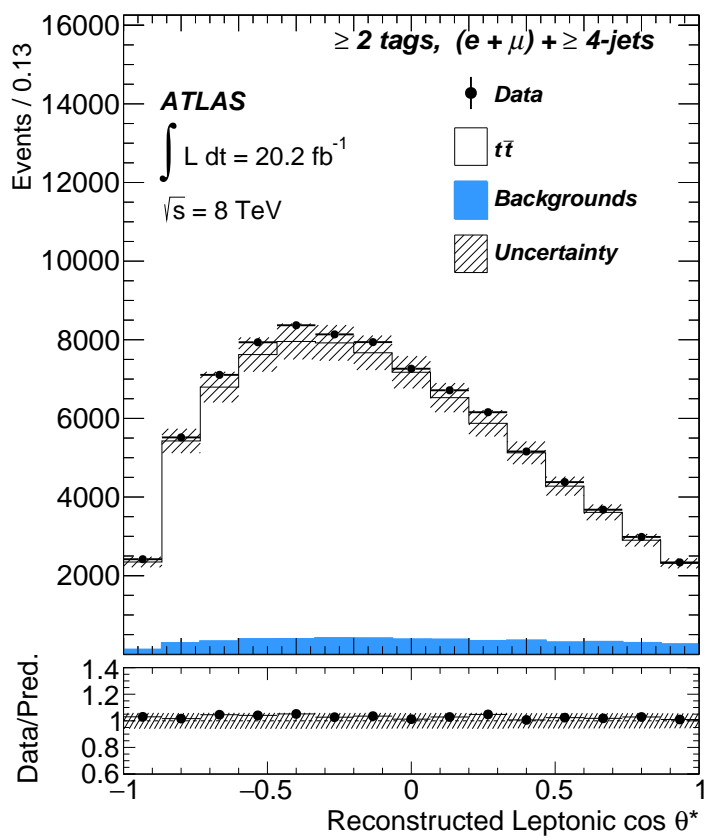

Figure 1: Measured and predicted $\cos \theta^{*}$ distribution for the leptonically decaying top quark. The dashed band corresponds to MC statistical and background normalization uncertainties. Figure from [4]

The ATLAS experiment performed the measurement of the $W$ helicity fractions using $20 \mathrm{fb}^{-1}$ of $\sqrt{s}=8 \mathrm{TeV}$ collision data in the $\ell+$ jets channel [4].

The helicity fractions are extracted from the angular distributions of the $W$ decay products through the normalized differential cross-section:

$$
\begin{array}{r}
\frac{1}{\sigma} \frac{d \sigma}{d \cos \theta^{*}}=\frac{3}{4}\left(1-\cos ^{2} \theta^{*}\right) F_{0} \\
+\frac{3}{8}\left(1-\cos \theta^{*}\right)^{2} F_{L}+\frac{3}{8}\left(1+\cos \theta^{*}\right)^{2} F_{R},
\end{array}
$$

where $\theta^{*}$ is the angle between the direction of flight of the $t$ quark in the $W$ rest frame and the direction of flight of the lepton or the $d$-type quark for the leptonic or hadronic $W$ decay, respectively. The reconstructed $\cos \theta^{*}$ distribution is in excellent agreement with the predictions, as shown in Figure 1 for the leptonic top. Templates correspondings to the $\cos \theta^{*}$ distributions for the three helicity component, and for both hadronic and leptonic top decays, are used to obtain the best description of the measured distributions, and to extract the helicity fractions. The measurement on the leptonic side is the most sensitive since the hadronic side is affected by a worse separation power and by larger uncertainties. The measured values are $F_{L}=0.299 \pm 0.015$, $F_{R}=-0.008 \pm 0.014$, and $F_{0}=0.709 \pm 0.019$. 
The structure of the $W t b$ vertex can be expressed in a general form introducing left- and right-handed (indicated with $L$ and $R$, respectively) vector $(V)$ and tensor ( $g$ ) couplings;

$$
\begin{gathered}
\mathcal{L}_{W t b}=-\frac{g}{\sqrt{2}} \bar{b} \gamma^{\mu}\left(V_{L} P_{L}+V_{R} P_{R}\right) t W_{\mu}^{-} \\
-\frac{g}{\sqrt{2}} \bar{b} \frac{i \sigma^{\mu v} q_{v}}{m_{W}}\left(g_{L} P_{L}+g_{R} P_{R}\right) t W_{\mu}^{-}+\text {h.c. },
\end{gathered}
$$

where $g$ is the weak coupling constant, $P_{L / R}$ represent the left- and right-handed chirality projection operators, and $m_{W}$ is the $W$ boson mass. In the SM all couplings vanish except $V_{L}$. Limits on anomalous coupling in the $W t b$ vertex, assumed to be real, are obtained from the measured helicity fractions by constraining the SM value $V_{L}=1$. The results are perfectly compatible with the SM and the $95 \%$ confidence level intervals for the anomalous couplings are: $[-0.24,0.31]$ for $V_{R}$, $[-0.14,0.11]$ for $g_{L},[-0.02,0.06]$ for $g_{R}$.

\section{4. $t \bar{t}$ production in association with a vector boson or a photon}

Measurements of the associated production of a $t \bar{t}$ pair with a vector boson or a photon allow to extract information about the coupling of the top quark with these particles, that could be altered in presence of physics beyond the SM.

\section{1. $t \bar{t}+Z$ and $t \bar{t}+W$}

The production cross-sections of $t \bar{t}$ with a massive vector boson $(t \bar{t}+V$, where $V$ can be either the $W$ or $Z$ boson) are predicted in the SM at the NLO in QCD to be $\sigma_{t \bar{t} Z}=0.84 \mathrm{pb}$ and $\sigma_{t \bar{t} W}=0.60 \mathrm{pb}$, with $12 \%$ uncertainty [5].

This section summarizes a recent ATLAS measurement of the $t \bar{t}+V$ production at $\sqrt{s}=13 \mathrm{TeV}$ using 3.2 $\mathrm{fb}^{-1}$ of collision data [6]. The event selection exploits the leptonic decays of top quarks and vector bosons to extract the signal from rare multi-lepton final state samples. The analysis strategy requires the determination of the event yields in several mutually exclusive event categories, such as two same-sign leptons (2L-SS), three leptons (3L) or four leptons (4L), some of which dominated by $t \bar{t}+W$, some by $t \bar{t}+Z$, others by backgrounds. These categories can be further classified depending on the number of $b$-jets. The determination of the fraction of the rare multi-lepton events originated by $t \bar{t}+V$ requires a careful evaluation of the event yields relative

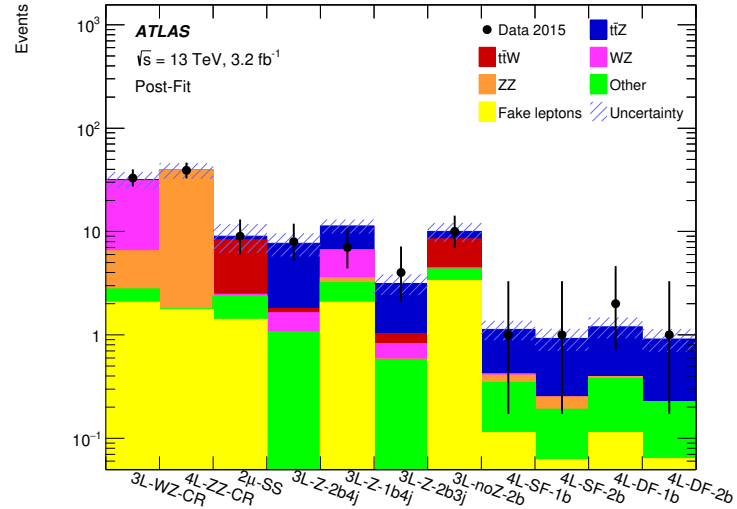

Figure 2: Measured and expected yields in all event categories used in the global fit to extract $\sigma_{t \bar{Z} Z}$ and $\sigma_{t \bar{t} W}$. The dashed band is the total uncertainty on the predictions. Figure from [6].

to all possible background sources, dominated by diboson production and events with fake-leptons. Background events containing well-identified isolated leptons are modeled using the MC simulation. The background yields of events containing at least one fake lepton are determined by defining appropriate control regions in collision data.

$\sigma_{t \bar{t} Z}$ and $\sigma_{t \bar{t} W}$ are extracted through a simultaneous fit of the event yields in all signal categories and two control regions dominated by di-boson production. Figure 2 shows the measured event yields and the expected ones after the fit procedure. The results are $\sigma_{t \bar{t} Z}=$ $0.92 \pm 0.29 \pm 0.10 \mathrm{pb}$ and $\sigma_{t \bar{t} W}=1.50 \pm 0.72 \pm 0.33 \mathrm{pb}$, in perfect agreement with the SM expectations and still dominated by the statistical uncertainty, leaving room for future improvements exploiting the large integrated luminosity that will be collected at the LHC.

\section{2. $t \bar{t}+\gamma$}

The production cross-sections of $t \bar{t}$ with a photon can be computed at the NLO in the SM. Anomalies in the production cross-section could point to modification of the $t-\gamma$ coupling that may reveal new physics effects.

The $t \bar{t}+\gamma$ production fiducial cross-section $\sigma_{t \bar{\gamma}}^{\mathrm{fid}}$ is measured by ATLAS using $20 \mathrm{fb}^{-1}$ of $\sqrt{s}=8 \mathrm{TeV}$ collision data [7]. A typical $t \bar{t}$ event selection in the $\ell+$ jets channel with the additional request of the existence of an isolated photon with transverse momentum $p_{\mathrm{T}}>15$ $\mathrm{GeV}$ is applied. The sample of selected events is composed by $t \bar{t} \gamma$, background events with a prompt $\gamma$ or with a fake $\gamma$. A template method is used to evaluate the different contributions to the total event yield. The variable $p_{\mathrm{T}}^{\text {iso }}$ defined as the sum of the $p_{\mathrm{T}}$ of all tracks within a 


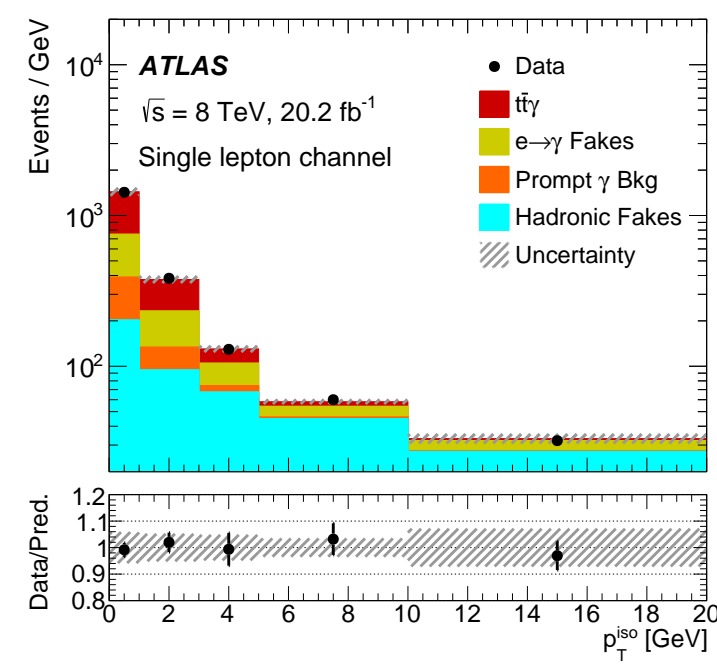

Figure 3: Post-fit $p_{\mathrm{T}}^{\text {iso }}$ distribution with the different contributions shown in different colors. The last bin includes all events with $p_{\mathrm{T}}^{\text {iso }}>20 \mathrm{GeV}$ and the dashed band is the total uncertainty on the predictions. Figure from [7].

cone of opening angle $0.2 \mathrm{rad}$ around the photon, is used to construct the templates, that for the $t \bar{t} \gamma$ signal and the prompt $\gamma$ are determined from the MC simulation while for the fake $\gamma$ backgrounds are evaluated on dedicated data control regions. Figure 3 shows the measured $p_{\mathrm{T}}^{\text {iso }}$ distribution with the various contributions determined after the fit with the templates.

The measured cross section is $\sigma_{t \bar{t} \gamma}^{\mathrm{fid}}=139 \pm 7 \pm 17$ fb. A dedicated calculation of $t \bar{t}+\gamma$ production fiducial cross-section at the NLO in QCD at $\sqrt{s}=8 \mathrm{TeV}$ has been carried out using the same technique as in Ref. [8], yielding $\sigma_{t \bar{t} \gamma}^{\text {fid }}=151 \pm 24 \mathrm{fb}$, in excellent agreement with the measured one. Fiducial differential cross-sections as a function of the transverse momentum and pseudorapidity of the photon have been measured, also showing excellent agreement with the SM predictions.

\section{Top quark mass measurements}

The mass of the top quark $m_{\text {top }}$ is a fundamental parameter of the SM and its precise determination is necessary to check the internal consistency of the SM [9]. Two recent ATLAS measurements of the top quark mass have been performed using $20 \mathrm{fb}^{-1}$ of $\sqrt{s}=8 \mathrm{TeV}$ collision data. The two measurements are done in the dilepton and the all-hadronic channel, selecting different event samples and being affected by different backgrounds and systematic uncertainties.
Both analyses use the template method, in which MC simulated distributions of a quantity particularly sensitive to $m_{\text {top }}$ are constructed, corresponding to a set of different $m_{\text {top }}$ values used in the event generator. These templates are modeled by analytical functions, whose parameters are determined as a function of $m_{\text {top }}$ and allow to interpolate between the templates. A likelihood fit of the measured distribution allows to determine the $m_{\text {top }}$ value that best describe the data. By construction the procedure is sensitive to the top mass used in the MC simulation, which can differ by up to approximately 1 $\mathrm{GeV}$ from the top quark pole mass [10].

\subsection{Top mass measurement in the dilepton channel}

The ATLAS measurement of $m_{\text {top }}$ in the dilepton channel is described in detail in Ref. [11]. Requirements in the $t \bar{t}$ dilepton channel event selection include the presence of two oppositely charged leptons, two or more jets, at least one of which $b$-tagged, allowing to select high purity samples, with total background contributions at the $1 \%$ level. The two jets with the highest value of the $b$-tagging discriminator algorithm are identified as top quark decay products and combined with the two leptons to compute the reconstructed lepton- $b$-jet invariant mass $m_{l b}^{\text {reco }}$, by choosing the combination with the lowest average invariant mass among the two possibilities. After an additional phasespace restriction, applied to optimize the total uncertainty, $m_{l b}^{\text {reco }}$ templates are constructed corresponding to $m_{\text {top }}=167.5,172.5,177.5 \mathrm{GeV}$.

Figure 4 shows the measured $m_{l b}^{\text {reco }}$ distribution with the best fit, corresponding to $m_{\text {top }}=172.99 \pm 0.41 \pm 0.74$ $\mathrm{GeV}$. The main sources of systematic uncertainties are the jet energy scale (JES) calibration $(0.54 \mathrm{GeV})$, the $b$ jet JES $(0.30 \mathrm{GeV})$, and the modeling of hadronization and initial- and final-state radiation $(0.23 \mathrm{GeV}$ each). A reduction of the systematic uncertainties is possible by exploiting the large samples that will be collected in LHC Run 2 to improve the calibrations and to restrict the phase-space of the measurement in regions with smaller uncertainties.

\subsection{Top mass measurement in the all-hadronic channel}

The measurement of $m_{\text {top }}$ in the all-hadronic channel is described in detail in Ref. [12]. Events with at least six jets, two of which $b$-tagged, satisfying additional selection requirements applied to improve the background rejection, are retained for the analysis. The $t \bar{t}$ final state is fully reconstructed among the possible combination of the six jets by means of a minimum $\chi^{2}$ approach based on the reconstructed invariant masses of the top 


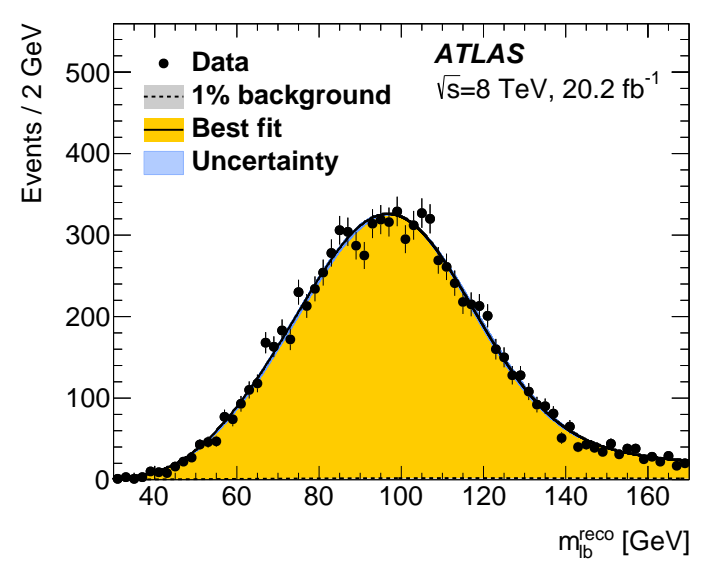

Figure 4: $m_{l b}^{\text {reco }}$ distribution shown for data with the fitted probability density function. The dashed line (barely visible at the bottom of the plot) indicates the background contribution. Figure from [11].

quarks and the $W$ bosons. The ratio of the 3 -jets to 2 -jets masses $R_{3 / 2}$ is used to extract the top quark mass. $R_{3 / 2}$ templates for the signal are obtained from MC by producing samples with $m_{\text {top }}=167.5,172.5,177.5 \mathrm{GeV}$ in the event generation phase. The template describing the multi-jet background distribution is determined using control regions on collision data. Figure 5 shows the $R_{3 / 2}$ distribution and the best fit, including signal and multi-jet background contributions, that corresponds to $m_{\text {top }}=173.72 \pm 0.55 \pm 1.01 \mathrm{GeV}$. The main sources of systematic uncertainty are the JES calibration $(0.60$ $\mathrm{GeV})$, the $b$-jet JES $(0.34 \mathrm{GeV})$, and the modeling of hadronization $(0.64 \mathrm{GeV})$.

Despite the differences among the dilepton and allhadronic channels, the size of the total uncertainty obtained on $m_{\text {top }}$ is similar and the two measurements can be considered as complementary.

\section{Summary}

The top quark provides the unique opportunity to study a "bare" quark and probe the theoretical predictions of its properties computed at the NLO or the NNLO. All top quark properties, measured using the data collected in LHC Run 1 and the first round of Run 2 , are in good agreement with the SM expectations. These measurements are used to improve the $t \bar{t}$ modeling in MC simulations and will have an impact on improving the background predictions for searches of new physics effects, in which the SM $t \bar{t}$ production often contributes to the background.

The huge increase of the available samples at LHC Run 2 offers unprecedented possibilities in top quark

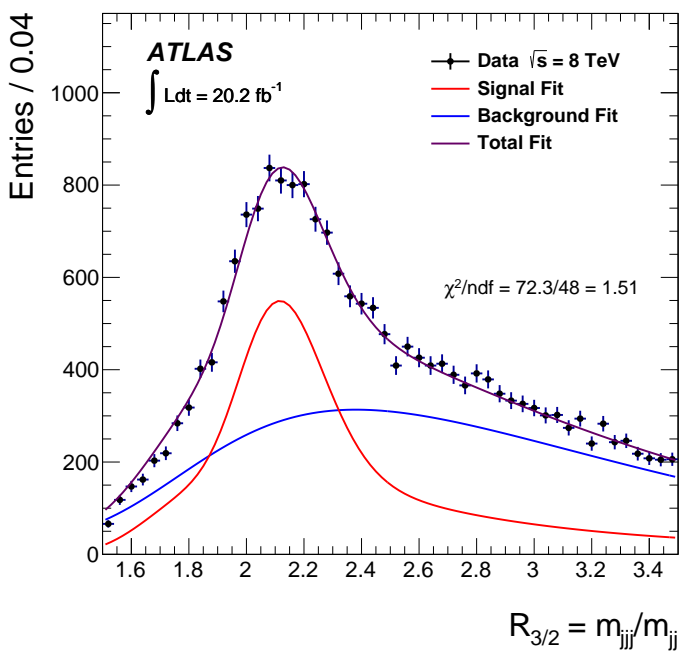

Figure 5: Measured $R_{3 / 2}$ distribution with the signal and multi-jet background contributions shown separately. Only the statistical uncertainty is shown. Figure from [12].

physics. The systematic uncertainties can be significantly reduced by improving the calibrations and focusing on restricted phase-space regions with smaller uncertainties. The aim is to reach an uncertainty on $m_{\text {top }}$ at the $\sim 300 \mathrm{MeV}$ level, as well as to improve the precision on measurements of top quark properties that potentially allow to observe deviations with respect to the SM expectations.

\section{References}

[1] ATLAS Collaboration, JINST 3 (2008) S08003.

[2] ATLAS Collaboration, JHEP 1703 (2017) 113.

[3] A. Czarnecki, J. G. Korner and J. H. Piclum, Phys. Rev. D 81 (2010) 111503

[4] ATLAS Collaboration, Eur. Phys. J. C 77 (2017) 264.

[5] S. Frixione, V. Hirschi, D. Pagani, H.-S. Shao and M. Zaro, JHEP 1506 (2015) 184

[6] ATLAS Collaboration, Eur. Phys. J. C 77 (2017) 40

[7] ATLAS Collaboration, arXiv:1706.03046 [hep-ex].

[8] K. Melnikov, M. Schulze and A. Scharf, Phys. Rev. D 83 (2011) 074013.

[9] M. Baak et al. [Gfitter Group], Eur. Phys. J. C 74 (2014) 3046.

[10] S. Moch et al., arXiv:1405.4781 [hep-ph].

[11] ATLAS Collaboration, Phys. Lett. B 761 (2016) 350.

[12] ATLAS Collaboration, arXiv:1702.07546 [hep-ex]. 